\title{
RELIABILITY OF RECHARGEABLE BATTERIES IN A PHOTOVOLTAIC POWER SUPPLY SYSTEM
}

\author{
Angel Urbina, Thomas L. Paez, Christian O'Gorman, Patrick Barney \\ Sandia National Laboratories \\ Experi:nental Structural Dynamics, 9119 \\ Mail Stop 0557 \\ Albuquerque, NM 87185-0557 \\ Rudolph G. Jungst, David Ingersoll \\ Sandia National Laboratories \\ Lithiunn Battery R\&D Department, 1521 \\ Mail Stop 0613 \\ A buquerque, NM 87185-0613
}

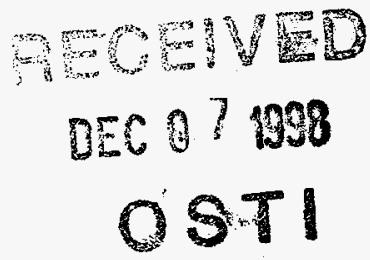

\begin{abstract}
We investigate the reliability of a rechargeable battery acting as the energy storage component in a photovoltaic power supply system. A model system was constructed for this that includes the solar resource, the photovoltaic power supply system, the rechargeable battery and a load. The solar resource and the system load are modeled as stochastic processes. The photovoltaic system and the rechargeable battery are modeled deterministically, ind an artificial neural network is incorporated into the model of the rechargeable battery to simulate darage that occurs during deep discharge cycles. The equations governing system behavior are solved simultaneously in the Monte Carlo framework and a first passage problem is solved to assess system reliability.
\end{abstract}

\section{Introduction}

A rechargeable battery energy storage system is an enabling component for making the power from a photovoltaic system dispatchable. A photovoltaic-based power supply system is sized to satisfactorily service the system load, and to charge the energy storage sub-system (typically a lead-acid battery), that will supply the system load when the photovoltaic system is not generating power. Optimal sizing of the system must account for the facts that the power generated by the photovoltaic system and, perhaps, the load as: well, are stochastic processes, and that prolonged deep discharge of the batteries diminishes their available capacity. A predictive capability for the performance of the battery under various system designs and operating options is needel to optimize the system for the best tradeoff among cost, load requirements, and battery life.

The present investigation extends and improves the techniques and results developed in a previous study (see Urbina et al., 1998). That study modeled the solar resource using a Markov chain, the photovoltaic system as deterministic, and incorporated an artificial neural network (ANN) to simulate damage in the battery caused by deep discharges-that is, 


\section{DISCLAIMER}

This report was; prepared as an account of work sponsored by an agency of the United States Government. Neither the United States Ciovernment nor any agency thereof, nor any of their employies, make any warranty, express or implied, or assumes any legal liability or responsibility for the accuracy, completeness, or usefulness of any information, apparatus, procluct, or process disclosed, or represents that its use would not infringe privately owned rights. Reference herein to any specific commercial product, process, or service by trade name, trademark, manufacturer, or otherwise does not necessarily constitute or imply its endorsement, recommendation, or favoring by the United States Governrnent or any agency thereof. The views and opinions of authors expressed herein do not necessarily state or reflect those of the United States Government or any agency thereof. 


\section{DISCLAIMER}

Portions of this document may be illegible in electronic image products. Images are produced from the best available original document. 
discharge of the battery below a threshold state-of-charge for a time duration beyond a threshold. It modeled the load as deterministic. The study performed probabilistic analysis in a Monte Carlo framework, and solved some preliminary problems in the probabilistic characterization of system behavior.

The present study extends the previous one in several ways. First, it incorporates a technique for estimation of the Markov chain transition probabilities (based on measured data) that is more accurate and much more efficient than the rudimentary technique used in the previous stucly. Second, it models the system load as a stochastic process. Clearly, any realistic analysis of system reliability must have the capability to incorporate random loads. Finally, the present study estimates system reliability via analysis of the first passage probability, below a failure threshold, of the maximum potential capacity of the rechargeable battery.

The following sections recapitulate sone of the fundamental assumptions and ideas of Urbina et al., (1998). The theory and algorithms that augment previous developments are presented in full. A numerical example that exercises the current developments is presented, and conclusions are offered.

\section{Mathematical Model of a Power Supply/S torage/Load System}

We develop a mathematical model and use it to simulate the operation of a renewable energy supply and storage system. The system model consists of four parts: a solar resource model, a photovoltaic power conversion model, a rechargeable battery energy storage model, and a load model. The following sections provide the details of these individual sub-models, then combine them into a model of the overall system. The solar resource is modeled as a random process, and we develop the capability to generate realizations of the solar radiation from the random source. These realizations are used as the system input, along with a load modeled as a stochastic process, which drives response in the battery storage system. Equations that permit simulation of battery behavior are solved for specific load and insolation inputs, and the results are used in a Monte Carlo framework to characterize system performance and reliability.

\section{The Solar Resource Model}

The first component to be modeled is the power source of the photovoltaic system: the sun. The amount of solar energy available on a given surface area is particularly important. This is the solar insolation, and it is measured in units of power per area-i.e. units of W-hr/ $\mathrm{m}^{2}$, BTU-hr' $\mathrm{ft}^{2}$, etc. There exist many sets of measured data that are readily available from local, state, and federal agencies. These data include values of global, direct and diffuse solar radiation (either measured with a pyranometer or calculated) and can be used to calculate the solar energy received by a solar collector (National Renewable Energy Laboratory, 1994), or to guide the development of an insolation model. A model was 
developed to calculate the solar energy received by a flat plate collector tilted at a specified angle and located at arbitrary latitude. Equation 1 identifies fundamental quanlities and their relation:

$$
E_{o}=E_{b c}+E_{d}+E_{g r}
$$

where $E_{o}$ is the total solar energy received by an inclined plane, $E_{b c}$ is the direct beam contribution, $E_{g r}$ is the ground reflected energy, and $E_{d}$ is the diffuse (or sky) energy (National Renewable Energy Laboratory's Analytic Studies Division, 1994). Each term along with its relation to more fundamental quantities is developed in the following.

The direct beam contribution $\left(E_{b c}\right)$ is de.ined by Equation (2):

$$
E_{b c}=E_{d n} \cos (\theta)
$$

where $E_{d n}$ is the direct normal solar radiation (hourly values available in databases) as measured with a pyranometer; it is defined as that radiation received within a $5.7^{\circ}$ fisld of view centered on the sun, and $\theta$ is the incident angle of the sun's rays to the collector (National Renewable Energy l_aboratory's Analytic Studies Division, 1994). The incident angle is a function of the latitude at which the collector i: located, its tilt angle and the position of the sun in the sky. A detailed explanation of how this value is obtained can be found in Kreider and Kreith, (1981).

The diffuse radiation model is describec by Equation (3):

$$
E_{d}=E_{d h}\left\{0.5\left(1-F_{1}\right)(1+\cos \beta)+F_{1}\left(\frac{a}{b}\right)+F_{2} \sin \beta\right\}
$$

where $E_{d}$ is the diffuse (sky) radiation, $E_{d h}$ is the diffuse horizontal solar radiation (hourly values available in databases) as measured with a pyranometer. $E_{d h}$ is defined as that radiation received from the sky (excluding the solar disk) on a horizontal surface, $F_{1}$ and $F_{2}$ are coefficients as sociated with the condition of the sky, a and $\mathrm{b}$ are functions of the position of the sun in the sky, and $\beta$ is the tilt angle of the collector (as measured from the horizontal). Equation (3) is also referred to as the Perez Model. A detailed explanation of its development and further discussion of the various parameters can be found in Perez et al., (1990).

The final component in Equation (1) is represented by Equation (4):

$$
E_{g r}=0.5 \rho E_{h}(1+\cos \beta)
$$

where $E_{g r}$ is the ground reflected radiation, $\rho$ is the surface reflectivity or albedo, and $E_{h}$ is the global horizontal solar radiation. $E_{h}$ is defined as the total radiation-i.e. direct and diffuse received on a horizontal surface, and $\beta$ is the tilt angle of the collector (from the horizontal). Albedo val גes range from 0.2 for green vegetation and some soils, 0.35 for old snow and up to 0.95 for dry new snow (National Renewable Energy Laboratory's Analytic Studies Division, 1994). 
Because the ground reflected radiation could be characterized as a function of the direct normal radiation and the diffuse horizontal radiation-that is, evaluating Eq. (2) at $\theta=0^{\circ}$ then adding $E_{d h}$ to it, gives $E_{h}$ which is ultimately used in Eq (4) to find $E_{g r}$, we model the latter two in the fcllowing.

\section{Synthetic Generation of Solar Data Using a V [arkov Process}

It was argued in Urbina et al., (1998) that the random variables representing direct normal solar radiation and diffuse horizontal radiation are dependent, and that their joint probability distribution is non-Gaussian. Substantial evidence confirming these arguments was presented. Because of this, a bivariate Markov chain was chosen to model the solar radiation phenomenon. It was explained that the Cartesian space of direct normal solar radiation and diffuse horizontal radiation can always be subdivided into a rectangular grid like the one shown in Figure 1. The data points are 310 realizations of direct solar radiation and diffuse horizontal radiation, and the individual rectangles group the radiation realizations into states of the Markov chain.

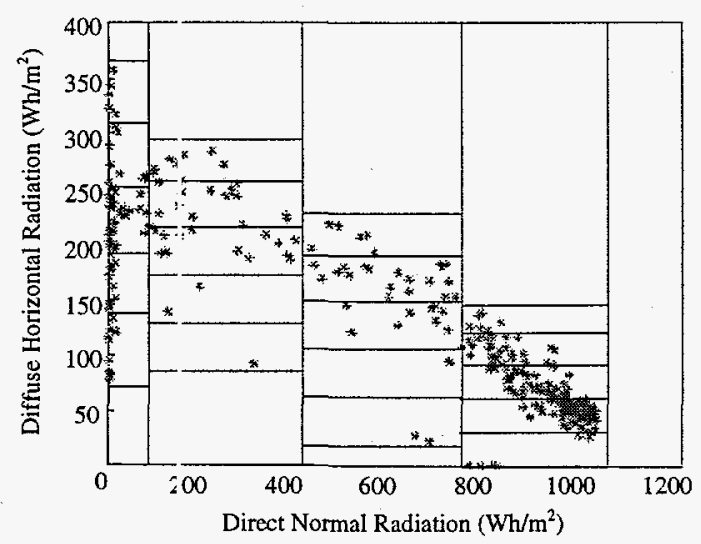

Figure 1: Cartesian space of direct normal and diffuse horizontal radiation

At any time, the solar radiation stochestic process occupies a state, and there is a probability mass function that describes the chance that each of the states is occupied. This is the state probability mass function, denoted $\boldsymbol{p}_{j}$, and defined as:

$$
p_{j}=\left\{\begin{array}{c}
P\left(X_{j}=s_{1}\right) \\
P\left(X_{j}=s_{2}\right) \\
\vdots \\
P\left(X_{j}=s_{N}\right)
\end{array}\right\} \quad j=0, \ldots, n-1
$$

where $P($.$) indicates the probability of the event described in parentheses, \boldsymbol{X}_{j}$ is a random variable in the random process $\left\{X_{j}, j=0, \ldots n-1\right\}$ that is the bivariate Markov chain, and the $s_{i}, i=1, \ldots, N$, are the system states. 
The probability, that the solar radiation random process state, transitions from $s_{i_{1}}$ at analysis time $j$, to $s_{i_{2}}$ at analysis time $j+1$ is given by the conditional probability mass function $P\left(X_{j+1}=s_{i_{2}} \mid X_{j}=s_{i_{1}}\right)$, and the collection of all these transition probabilities is the transition malrix:

$$
\begin{aligned}
& P_{j+1 \mid j}=\left[\begin{array}{cccc}
P\left(X_{j+1}=s_{1} \mid X_{j}=s_{1}\right) & P\left(X_{j+1}=s_{1} \mid X_{j}=s_{2}\right) & \ldots & P\left(X_{j+1}=s_{1} \mid X_{j}=s_{N}\right) \\
P\left(X_{j+1}=s_{2} \mid X_{j}=s_{1}\right) & P\left(X_{j+1}=s_{2} \mid X_{j}=s_{2}\right) & \ldots & P\left(X_{j+1}=s_{2} \mid X_{j}=s_{N}\right) \\
\vdots & \vdots & \ddots & \vdots \\
P\left(X_{j+1}=s_{N} \mid X_{j}=s_{1}\right) & P\left(X_{j+1}=s_{N} \mid X_{j}=s_{2}\right) & \cdots & P\left(X_{j+1}=s_{N} \mid X_{j}=s_{N}\right)
\end{array}\right] \\
& j=0, \ldots, n-2
\end{aligned}
$$

This transition probability matrix and the state probability mass function at time zero are sufficient to establish the probability mass functions for states at all times because of the following relation:

$$
\boldsymbol{p}_{j+1}=\boldsymbol{P}_{j+1 \mid j} \boldsymbol{p}_{j} \quad j=0, \ldots, n-2
$$

Because the solar radiation random process čn be started at night when radiation is certain to be zero, $\boldsymbol{p}_{0}$ is trivially specified. Though the method for obtaining the transition probabilities from measured data was not detailed in Urbina et al., (1998), the technique was simple and direct, but not very efficient. We outline in the following a much improved technique. Our objective is to use measured solar radiation data to estimate the transition probability matrices of Equation (6). Let the data representing realizations of the system state at a particular hour, and during a particular month, and the corresponding data measured during the following hour be denoted by $\boldsymbol{x}_{j}, \boldsymbol{y}_{j}, j=1, \ldots m$, respectively. If data measured over a period of 10 years are used here, and the month under consideration is January, then $m=310$. For example the $x_{j}$ might be two-element vectors; the first element represen s the direct normal radiation and the second element represents the diffuse horizontal radiation at 9:00 AM on a January day. The $y_{j}$ represent the same quantities one hour later. (We assume that the data have been normalized prior to this analysi s.) The joint probability density function (PDF) of the source of these two dependent quantities can be approximated using the kernel density estimator (KDE):

$$
\hat{f}_{x y}(x, y)=\frac{1}{\sqrt{2 \pi \varepsilon}} \sum_{j=1}^{m} \Phi\left(\frac{y-y_{j}}{\varepsilon}\right) \exp \left(-\frac{1}{2 \varepsilon^{2}}\left(x-x_{j}\right)^{2}\right)
$$

For details on the KDE see Silverman, (1986). Likewise, the marginal PDF of the random variable that is the source of the $\boldsymbol{x}_{j}, j=0, \ldots, m$, can be approximated with the $\mathrm{KDE}:$

$$
\hat{f}_{x}(x)=\frac{1}{\sqrt{2 \pi \varepsilon}} \sum_{j=1}^{m} \exp \left(-\frac{1}{2 \varepsilon^{2}}\left(x-x_{j}\right)^{2}\right) \quad-\infty<x<\infty
$$


Now, the conditional PDF of the random variable $y$ given the random variable $\boldsymbol{x}$ is defined as:

$$
f_{(y \mid x)}(y \quad x)=\frac{f_{x y}(x, y)}{f_{x}(x)} \quad-\infty<y<\infty
$$

And the conditional cumulative distribution function (CDF) of $y$ given $\boldsymbol{x}$ can be obtained by integrating the conditional PDF:

$$
F_{(y \mid x)}(y \mid x)=\int_{-\infty}^{y} f_{y \mid x}(\theta \mid x) d \theta \quad-\infty<y<\infty
$$

In view of this, the KDEs of Equations (8) and (9) can be used to approximate the conditional CDF of $\boldsymbol{y}$ given $\boldsymbol{x}$. It is:

$$
\hat{F}_{y \mid x}(y \mid x)=\frac{\sum_{j=1}^{m} \Phi\left(\frac{y-y_{j}}{\varepsilon}\right) \exp \left(-\frac{1}{2 \varepsilon^{2}}\left(x-x_{j}\right)^{2}\right)}{\sum_{j=}^{m} \exp \left(-\frac{1}{2 \varepsilon^{2}}\left(x-x_{j}\right)^{2}\right)} \quad-\infty<y<\infty
$$

where $\Phi($.$) is the CDF of the standard normal random variable, and \varepsilon$ is the smoothing factor of the KDEs. This approach can be used, along with the definition of the state boundaries to approximate the transition probability matrices.

As in the previous study, we use the Markov chain model in a Monte Carlo framework to generate as many realizations of the random process as desired.

\section{The Photovoltaic Power Supply Model}

The power to this system is supplied by a photovoltaic array that transforms the solar insolation into dc current at a specified voltage. Because of the complex be iavior of these polycrystalline silicon arrays, a number of models have been proposed to relate the insolation input to the expected output of the arrays. Some of these models take into account the cell's physical characteristics-i.e. temperature, chemical composition, etc.-and the location's ambient conditions. For this project, a specific type of photovoltaic array was selected and its maximum rated current output was used to determine the current produced at a particular time. In other words, given the maximum rated current of the photovoltaic array, $I_{m p}$, (which we will consider a constant) and the sol ar insolation, $E_{0}(\mathrm{t})$ at time $\mathrm{t}$, the current produced by the array is given by

$$
I(t)=I_{m p} \cdot E_{0}(t) / C_{0}
$$

where $\mathrm{C}_{0}$ is the energy per square meter applie $\mathrm{d}$ to the collector over a computational time increment, at which the current $I_{m p}$ is generated. A time history of hourly current values can be obtained by using the output of Eq. (1) in Eq. (13). 


\section{The Load Model}

The load model appropriate to the analysis of a particular system depends on the particular use that the photovoltaic power supply system is intended to serve. The current demanded by the load at a specified voltage is denoted $I_{L}(t)$ and we choose to introduce the potential for random loads in this investigation. During nighttime hours the load is modeled as constant, $I_{L_{1}}$. During daylight hours, the load is modeled as a uniformly distributed, band-limited, white noise random process. That is:

$$
I_{L}(t)=I_{\left.L_{0}\right)} \cdot w(t)
$$

where $I_{L_{0}}$ is a constant (for this project, this value was taken as $75 \%$ of the maximum $I_{L_{1}}$ ), and $w(t)$ is a uniformly distributed, band-limited, white noise random process with values in $[0,1]$.

\section{The Rechargeable Battery Model}

The focus of this investigation is to characterize the reliability of rechargeable batteries, which are recharged from a renewable source that provides power in random increments. Further, we narrow our focus more by concentrating on the damage that can accumulate in rechargeable tatteries, specifically lead-acid batteries, when they are subjected to deep discharge use cycles. In particular, it is known that when lead-acid batteries are used at a low state of charge, the maximum potential capacity can be significantly diminished. This may eventually lead to battery failure. In view of this, we develop a framework to model battery state of charge and maximum potential battery capacity as functions of time. We introduce the damage effect that occurs during deep distharge via a non-positive function of deep discharge duration and depth. Because the form of this function is unknown, we model it with an artificial neural network (ANN) whose parameters are to be trained with experimental data. (This training has not yet been accomplished, so for now the ANN model has been trained with data we consider plausible.) O'Gorman et al., (1998) first demonstrated the use of ANNs to simulate battery performance.

We introduce notation for the current demanded from the battery, $I_{B}(t)$, and the recharge current available to the battery, $I_{R}(t)$, for use in the development of the system's governing equations. In terms of these quantities, the state of charge in the rechargeable battery system can be expressed:

$$
C(t)=\left\{\begin{array}{l}
\int_{t_{1}}^{t} \gamma(\tau)\left[I_{R}(\tau)-I_{B}(\tau)\right] d \tau+C\left(t_{0}\right) \quad t \geq t_{0}, \quad C(t) \leq M_{C}(t) \\
M_{C}(t) \quad \text { otherwise }
\end{array}\right.
$$


The function $M_{C}(t)$ is the maximum potential battery capacity at time $t$. The function $\gamma(t)$ is the recharge efficiency that establishes the effective rate at which recharge san occur. In general, as a rechargeable battery nears its maximum potential capacity $\gamma(t)$ approaches zero, and at lower lievels $\gamma(t)$ is near one. Battery testing would be required to establish the specific form and parameters of this function. For present purposes we arbitrarily take the function to be:

$$
\gamma(t)=\left\{\begin{array}{l}
1 \quad c(t) / M_{C}(t)<\alpha, \quad \alpha \in(0,1) \\
0.5\left[1-\cos \pi\left(1+\frac{\left(C / M_{C}\right)-\alpha}{1-\alpha}\right)\right] \quad \alpha \leq C(t) / M_{C}(t) \leq 1
\end{array}\right.
$$

Because the function $M_{C}(t)$ tracks the maximum potential battery capacity as a function of time and because we take damage to a rechargeable lead-acid battery (when used in photovoltaic application) caused by deep discharge as irreversible, $M_{C}(t)$ must be a monotone non-increasing function. We choose the following as the form for $M_{C}(t)$ :

$$
M_{C}(i)=\int_{t_{0}}^{t} \delta_{M}(\tau) d \tau+M_{C}\left(t_{0}\right) \quad t \geq t_{0}
$$

The function $\delta_{M}(t)$ must be non-positive and must indirectly characterize damage to the maximum potential capacity of the rechargeable battery during deep discharge. F(r present purposes we assume that $\delta_{M}(t)$ is a function of the time duration of a discharge beyond a threshold, $T(\mathrm{t})$, and the depth of discharge above a threshold, $D(t)$. Therefore, we write

$$
\delta_{M}(t)=g_{\delta}(T, D)
$$

We specify that $g_{8}(T, D)$ is zero for $T(t)$ below its threshold value or $D(t)$ below its threshold value, but beyond this we do not know the form of $g_{\delta}(T, D)$. It is anticipated that an explicit form for $g_{\delta}(T, D)$ cannot be easily derived, so we choose to model the function using an ANN. Any of a number of robust forms can be used here including, for example the layered perceptron ANN (see Freeman and Skapura, 1991), or the radial basis function ANN (see Moody and Darken, 1989). In the numerical example to follow, wis use a particular form of the layered perceptron ANN to model $g_{\delta}(T, D)$. The advantage in use of an ANN here is that given sufficient training data, it learns rapidly. Further, ANNs are accurate and efficient. The ANN used to model $g_{\delta}(T, D)$ ir this investigation is shown in Figure 2. This model will be only applicable to the particular type of battery used in this proj zct.

There are two practical issues that nust be resolved before the equations governing battery behavior can be implemented. First, as deep discharge of the rechargeable battery occurs, a smoothing must be applied to the state of charge in order for sensible realizations of the function $g_{\delta}(T, D)$ to be modeled. We choose to apply a one-day moving average of the state of charge in our definition of $D(t)$. Second, we seek to keep track of the maximum potential capacity of the battery at all times; however, when the system is in the midst of a deep discharge cycle, the ultimate duration of the cycle is 
unknown. To accommodate this fact, once a deep discharge cycle has commenced, we estimate its ultimate duration by noting the present state of the system and assuning that the state of charge will recover at the average rate of recharge.

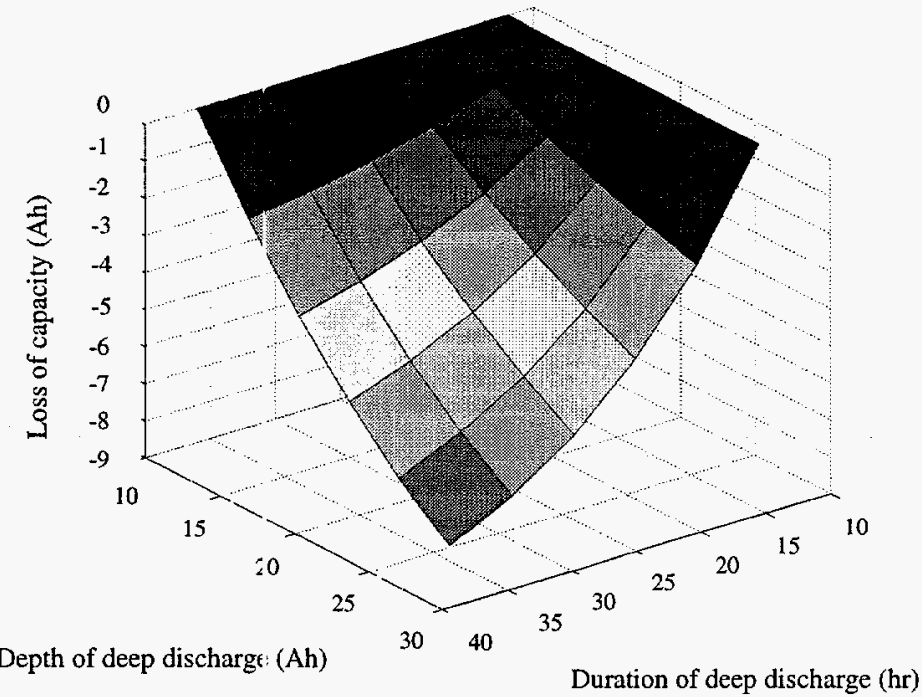

Figure 2: ANN model of battery damage

\section{Auxiliary Equations}

Two additional equations relating photovoltaic production and load demand, to battery recharge and discharge are required to simulate the behavior of the overall system. Previously, $I_{L}(t)$ was defined as the current demand at the load, $I_{P V}(t)$ was defined as the current available from the photovoltaic system, and $I_{B}(t)$ was defined as the current demand on the battery. These quantities are related via Equation (19):

$$
I_{B}(t)=H\left[I_{L}(t)-I_{P V}(t)\right]\left(I_{L}(t)-I_{P V}(t)\right)
$$

where $H[$.$] is the Heaviside unit step function. This indicates that the current demand on the battery is a linear function of$ the excess of the load demand beyond capabilily of the photovoltaic system to supply it. The current demand on the battery is zero when the load does not exceed the photovoltaic supply.

The quantity $I_{R}(t)$ was defined as the recharge current available to the battery. It is expressed:

$$
I_{R}(t)=H\left[I_{P V}(t)-I_{L}(t)\right]\left(I_{P V}(t)-I_{L}(t)\right)
$$

It is proportional to the excess of the photovoltaic system supply beyond the load demand, when this quantity is positive, and zero when this quantity is negative.

We combine Eqs. (19) and (20) witt. Eqs. (1) through (7) describing the solar resource, Eq. (13) and Eq. (14) describing the photovoltaic power supply and the load respectively, and Eqs. (15) through (18) describing battery behavior, to simulate the system. The following numerical example shows the results of some simulations. 


\section{Numerical Example}

Because the current analyses are being run in the Monte Carlo framework, any of a number of probabilistic characterizations of system behavior can be developed. Since we are interested in performing reliability analysis, we choose to perform a first passage probability analysis on the response. The first passage probability distribution of a random process is the chance that the random process passes beyond a barrier of interest, for the first time, at or before a time $t$. Here, we will estimate the first passage probability of $M_{v}(t)$, the maximum potential capacity, below a barrier that is a fraction of its initial value. The first passage problem is defined as follows. Let $\left\{Y_{j}, j=0, \ldots, n-1\right\}$ be a random process, with discrete time index $j$, and let $T_{s}$ be a random variable clenoting the first time index at which the state of the random process passes into the set $S$. Then the CDF of the random variable $T_{S}$ is the first passage probability distribution of the random process $\left\{Y_{j}, j=0, \ldots, n-1\right\}$, with reference to the set of states $S$.

We estimate the $\mathrm{CDF}$ of the random variable $T_{S}$ using the realizations of $\left\{Y_{j}, j=0, \ldots, n-1\right\}$ generated during the Monte Carlo analysis. Denote these realization: $y_{j}^{(k)}, j=0, \ldots, n-1, k=1, \ldots, M$, where $M$ is the total number of realizations generated during the Monte Carlo analysis. We estimate, for each realization, the time at which the signal first passes into the set $S$. (We accomplish this, for example, simply by tracking the response time history.) Denote by $t^{(k)}, k=1, \ldots, M$, the time at which the $(\mathrm{k})^{\text {th }}$ realization passes into the state $S$. (Some realizations may not pass into the state $S$, within the time indices $j=0, \ldots, n-1$. For these, the quantity $t^{(k)}$, might be set to some value that is large relative to the time index $j=n$. For such a case, the first passage probability distribution will not reach a value of one by $j=n-1$.) The ensemble of values $t^{(k)}, k=1, \ldots, M$ can now be used in the kernel cumulative distribution function estimator to approximate the CDF of the random variable $T_{S}$. This is:

$$
F_{T_{S}}(t)=\frac{1}{M} \sum_{k=1}^{M} \Phi\left(\frac{t-t^{(k)}}{\varepsilon}\right)
$$

where $\Phi($.$) is the standard normal CDF, and \varepsilon$ is the smoothing factor of the kernel CDF estimator.

The first test case to be analyzed here (denoted as system \#1) is based on an insolation model developed from data measured in Albuquerque, New Mexico (latitude $\sim 35^{\circ}$ North). It assumes the use of a non-concentrating, non-tracking array, tilted at an angle equal to the latitude. Each module of the array is rated at 2.3 amps (at standard test conditions) and three modules are connected in parallel for a total of 6.9 amps. The system includes a rechargeable battery rated at 12 volts and $105 \mathrm{Ah}$ (which is $\mathrm{M}_{\mathrm{c}}\left(\mathrm{t}_{0}\right)$ in Eq. 17) and a constant, nocturnal load of one 18-watt lamp with an operational current of 1.9 amps and a random daytime load calculated with Eq. (14). This combination applies an average load of $\sim 30$ Ah per day. 
One hundred one-year long simulations were ginnerated and analyzed using these parameters. First passage probability distributions are estimated for maximum potential capacity values that are $99,95,85$ and 75 percent of the initial maximum potential capacity.

Because the first passage probability distribution is a CDF, it is monotone non-decreasing. This characteristic is reflected in the graph of Figure 3. However, it may appear peculiar that there is much increase at the beginning of the curve, then later, at the end of the curve. The reason is that the analysis was started on January 1 and the collector was tilted at an angle that maximizes the yearly energy output. The weather during that time of year tends to be cloudy, and batteries tend to operate in a state of deep discharge, yielding an increased probability of damage. The weather late in the calendar year also tends to be cloudy, again, leading to battery damage. This is the reason for the increase at the end of the graph. If we change the parameters of the analysis (such as the tilt angle of the collector), the results will differ. The effect of changing the tilt angle is shown in Figure 4. In system \#2 the tilt angle was changed to $50^{\circ}$ (which is the latitude angle plus $15^{\circ}$ ). This change skews the energy production toward the vinter months. The other parameters are left unchanged. It can be seen from the figure that most batteries survived (with out damage) the cloudy weather in January. In addition it is noted that a small percentage $(\sim 6 \%)$ of the cases, survived the entire year with a capacity of $99 \%$ (of their initial maximum potential) or higher.

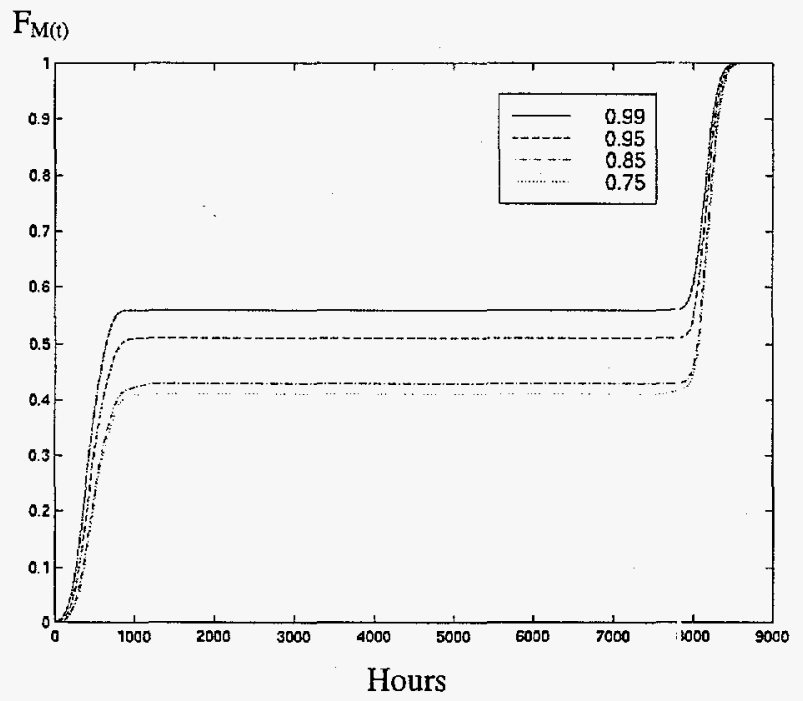

Figure 3: CDF for system \#1

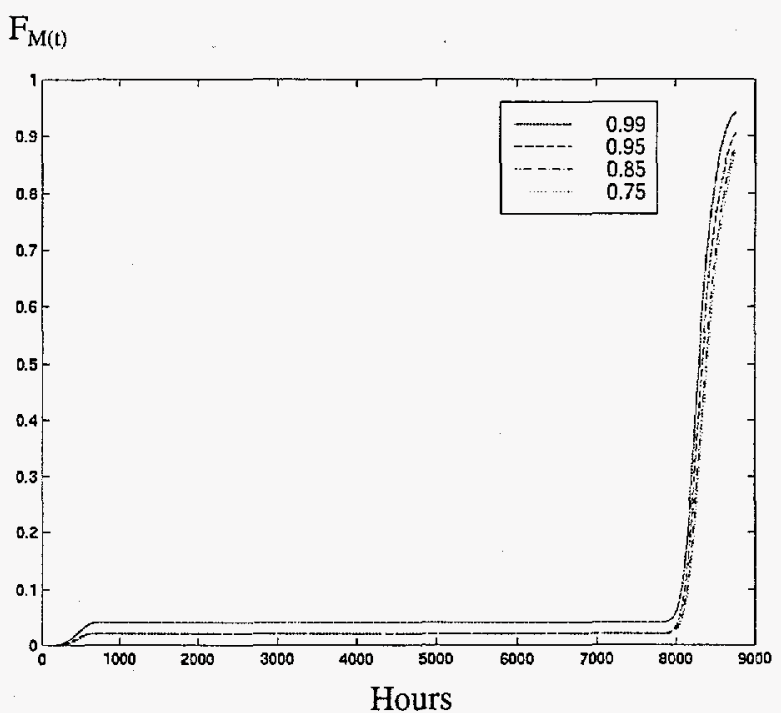

Figure 4: CDF for system \#2

\section{Conclusions}

A technique for the analysis of reliability of rechargeable batteries in photovoltaic power supply systems was developed. The present analysis introduces several improvements over previous studies. Among these is an accurate and efficient technique for the estimation of transition probabilities of the Markov chain used to characterize the battery state of 
charge and maximum potential capacity. Beyond this, a stochastic load was introduced into the model, and a first passage probability analysis was introduced into the analysis of the results. The parameters of the model developed here can be adjusted to investigate a wide variety of systems, yielding practical reliability analyses of rechargeable batteries in a photovoltaic power supply system.

The artificial neural network used $: 0$ characterize damage in the rechargeable battery, and developed in the previous study, was used again in this investigation. Experiments remain to be performed that specify the precise parameters of the neural network. Further, a worthwhile : goal of future investigations is the transition of the current analysis from the Monte Carlo framework to a direct analysis framework.

\section{Acknowledgment}

Sandia National Laboratories is a multiprogram laboratory operated by Sandia Corporation, a Lockheed Martin Company, for the United States Department of Energy under Contract DE-AC04-94AL85000.

\section{References}

J. A. Freeman and D. M. Skapura, Neural Networks, Algorithms, Applications, and Programming Techniques, AddisonWesley Publishing Company, Reading, Massachusetts, (1991).

D. Isaacson and Madsen, R., Markov Chains: Theory and Applications, Wiley, New York, (1976).

J. F. Kreider and F. Kreith, Solar Energy Handbook, McGraw Hill, New York, 1981, pp. 2-1 - 2-18 and 2-72 - 2-73.

J. Moody and C. Darken, "Fast Learning in Jetworks of Locally-Tuned Processing Units," Neural Computation, (1) 2, (1989), pp. 281-294.

National Renewable Energy Laboratory, National Solar Radiation Database User's Manual (1961-1990), version 1.1, (1994).

National Renewable Energy Laboratory's An:lytic Studies Division, Solar Radiation Data Manual for Flat-Plate and Concentrating Collectors, (1994).

C. C. O'Gorman, D. Ingersoll, R. G. Jungst and T. L. Paez, Artificial Neural Network Simulation of Battery Performance, Proceedings of the thirty-first Hawaii International Conference on System Sciences, IEEE, The University of Hawaii, Hawaii, Hawaii, (1998).

R. Perez, P. Ineichen, R. Seals, J. Michalsky and R. Stewart, Modeling Daylight Availability and Irradiance Components from Direct and Global Irradiance, Solar Energy, Vol. 44, No. 5, 1990, pp. 271-289.

B. Silverman, Density Estimation for Statistics and Data Analysis, Chapman \& Hall, Monographs on Statistics and Applied Probability 26, London, (1986).

A. Urbina, D. Ingersoll, R. G. Jungst, T. L. Paez, C. C. O'Gorman and P. Barney, Probabilistic Analysis of Rechargeable Batteries in a Photovoltaic Power Supply System, Proceedings of the $194^{\text {th }}$ Electrochemical Society Meeting, Boston, Massachusetts, (1998). 